\title{
RING DIAGRAMS AND ELECTROWEAK PHASE TRANSITION IN A MAGNETIC FIELD
}

\author{
Vladimir Skalozub $^{a}$ and Michael Bordag ${ }^{b}$ \\ ${ }^{a}$ Dniepropetrovsk University, Dniepropetrovsk 320625, Ukraine \\ e-mail: skalozub@ff.dsu.dp.ua \\ ${ }^{b}$ Institut für Theoretische Physik, Leipzig University, 04109, Leipzig, \\ Germany, e-mail: Michael.Bordag@itp.uni-leipzig.de
}

PACS Numbers: 11.10.Wx, 11.15.Ex

\begin{abstract}
Electroweak phase transition in a magnetic field is investigated within the one-loop and ring diagram contributions to the effective potential in the minimal Standard Model. All fundamental fermions and bosons are included with their actual values of masses and the Higgs boson mass is considered in the range $75 \mathrm{GeV} \leq m_{H} \leq 115 \mathrm{GeV}$. The effective potential is real at sufficiently high temperature. The important role of fermions and $W$-bosons in symmetry behaviour is observed. It is found that the phase transition for the field strengths $10^{23}-10^{24} \mathrm{G}$ is of first order but the baryogenesis condition is not satisfied. The comparison with the hypermagnetic field case is done.
\end{abstract}

1. The concept of symmetry restoration at high temperature has been intensively used in studying the evolution of the universe at its early stages. Nowadays it gives a possibility to investigate various problems of cosmology and particle physics [1], [2]. In particular, the type of the electroweak phase transition and hence the further evolution of the universe depend on the Higgs boson mass $m_{H}$. Most investigations of the electroweak (EW) phase transition have included into consideration high temperature as the main environment [2], [3]. But in recent years cogent arguments following from different approaches in favour of the presence of strong magnetic fields at that stage have appeared [5], [6] (for recent review see [7]). So, the phase transition at high temperature and strong fields has to be of interest. Moreover, at present time when masses of all fundamental particles, except $m_{H}$, are known it is desirable to investigate in details the phase transition as the function of this parameter. 
One of the possibilities to have strong magnetic fields in the EW phase transition epoch was discussed by Vachaspati [5]. From his analysis it follows that under very general conditions the fields $H \sim T_{i}^{2}$ in the patches of sub-horizon scales can be generated during a large class of grand unified transitions [6], [7], where $T_{i}$ is transition temperature. The second one is the formation of the Savvidy vacuum magnetic state at high temperature ( $H \sim g T^{2}, g$ is gauge coupling constant) [10, 20, 21, 23]. In latter case only the abelian field configurations could arise spontaneously since they are sourceless. For many problems of cosmology it is important to estimate the field strengths presented, but it is difficult to realise that without detailed investigations within specific models. Usually, only one type of fields is considered. Therefore, results obtained in such a way give an upper estimate of the field. This remark is relevant to our analysis.

Various aspects of the phase transitions in magnetic fields at high temperature have been investigated by many authors [20]-[28]. In Ref. [22] the influence of magnetic field on the sphalerons and the possible consequences of that for the EW phase transition were considered. These studies are concentrated mainly on the influence of the boson fields only. But due to a rather heavy $t$-quark mass, $m_{t} \simeq 175 \mathrm{GeV}$, the influence of fermion sector increases. This is the case in strong magnetic fields even at low temperature due to the presence in the one-loop effective potential (EP) of the term $\sim m_{f}^{2} e H$, where $m_{f}$ is fermion mass. Moreover, at high temperature not only heavy but also light fermions are important, as it follows from the term $\sim H^{2} \log T / m_{f}$ of the EP which significantly influences the EP curve in the broken phase. Actually, it will be shown below that the strong magnetic fields affect essentially the phase transition dynamics. Another aspect of the phase transition, which also was not investigated but plays an important role, is the influence of the so-called ring diagrams at high temperature and strong field. At zero field it was considered in Refs. 24, [25] where their importance for determining of the type of the phase transition has been shown. In the latter paper the t-quark mass was taken of order $110 \mathrm{Gev}$. So, taking into account present day data, it should be considered as a qualitative estimate of the effect of ring diagrams even for zero-field case.

The aim of the present paper is to investigate the EW phase transition at high temperature and constant strong magnetic fields $H$. We consider the case when the magnetic field is present in both, the restored and broken phases. This scenario may be realized in nature when the field is generated due to the Savvidy mechanism at a GUT scale. We calculate and investigate 
the one-loop EP and the contributions of ring diagrams. We include all bosons and fermions with their actual masses. So, the mass $m_{H}$ remains the only free parameter. Taking into account the present day experimental limit $m_{H} \geq 90 \mathrm{GeV}$, we consider the range of the mass values $75 \mathrm{GeV} \leq m_{H} \leq$ $115 \mathrm{GeV}$. The lower bound chosen corresponds to the values of the mass when perturbative methods give reliable results. For heavier masses it should be considered as an estimate. But we belive that the effects of strong fields being included exactly are not very sensitive to the change of the mass range investigated. With these approximation adopted we observed that for weak magnetic fields the phase transition is of second order or of weak first order. The increase of the field strength make it stronger first order. But even for the field strengths $H \sim 10^{23}-10^{24} G$ the baryogenesis does not survive in the minimal Standard Model (SM).

It will be important for what follows to remember recent results on obeservation of the gluon magnetic mass in lattice simulations that was found to be of order $m_{\text {mag }} \sim g^{2} T$ (as has been expected from nonperturbative calculatios in quantum field theory [11], [12]). The mass screens the nonabelian component of magnetic fields at distances $l>l_{m} \sim\left(g^{2} T\right)^{-1}$ but inside the space region $l<l_{m}$ it may exist. Since the typical order of particle masses at high temperature is $M \sim g T$, the spectrum of charged particles is formed at the space range of Larmor's radius $r_{L} \sim(g H)^{-1 / 2}$ and the magnetic field strength generated at high temperature has the order $(g H)^{1 / 2} \sim g^{2} T$ [20], [23] the field is able to affect all the processes at high temperatures. The latter fact was not taken into account in a number of investigations of the EW phase transition. In particular, in recent papers [13, 14] ( as in Ref. [21]) the field strength generated at finite temperature was erroneously estimated as coinciding with that at zero temperature. Hence, it has been concluded that the magnetic fields could not be spontaneously generated at all (because for weak fields generated in the vacuum the Larmor radius is larger then the inverse magnetic mass and such fields must be screened).

In papers [16], [17], [18 the influence of the hypermagnetic field on the EW phase transition has been investigated. In Ref. [16] the EP was computed in a tree approximation and the result that the presence of $H_{Y}$ makes the week first-order phase transition stronger has been derived. In Ref. [17] the temperature dependent part of the EP was calculated in one-loop order whereas the field has also been taking into account at tree level. By investigating the EP these authors came to the conclusion that the hypermagnetic field induces strongly first order EW phase transition. Moreover, 
they found that for $H_{Y}>0.3-0.5 T^{2}$, where $T$ is the transition temperature, the standard baryogenesis survives. However, we would like to note that for the week first-order phase transition the fluctuations are essential, the one-loop approximation to the EP is not sufficient and the correlation corrections must be included [25], [11]. In Refs. [18], 19] this problem has been investigated by lattice simulations. We will compare that results with the ones presented here in the last section.

To make a link between studies of symmetry behaviour in external hypermagnetic field and previous results for the case of usual magnetic field [28], 20] we note that in the broken phase $H_{Y}$ and $H$ are connected by the relation $H=H_{Y} \cos \theta_{w}$, where $\theta_{w}$ is the Weinberg angle. So, all investigations dealing with symmetry behaviour in a magnetic field at high temperature are relevant to the case of $H_{Y}$ in the respect of the form of the EP curve at different $T, H_{Y}$. The hypercharge field influences the scalar field condensate at tree level and acts to restore symmetry. That was the reason why it has been taken into account in the lowest order. But, as it will be shown below, for strong fields and heavy $m_{H}$ the form of the EP curve in the broken phase is very sensitive to the change of the parameters. Moreover, it is strongly dependet on the correlation correction contributions of heavy particles. So, to have an adequate picture of the EW phase transition the symmetry behaviour with rings included has to be investigated.

2. The Standard Model Lagrangian is well known (see, for example, Refs. [2], [33]). The one-loop contributions of bosons and fermions to the EP at finite temperature and magnetic field have been calculated and for detais we refer readers to papers [28], [33], [20], [32]. Below, we consider only some necessary information about that and concentrate our attention on calculation of the ring diagrams.

The external electromagnetic field is introduced by splitting the potential in two parts: $A_{\mu}=\bar{A}_{\mu}+A_{\mu}^{R}$, where $A^{R}$ describes a radiation field and $\bar{A}=\left(0,0, H x^{1}, 0\right)$ corresponds to the constant magnetic field directed along the third axis. We make use of the gauge-fixing conditions [33]

$$
\begin{aligned}
\partial_{\mu} W^{ \pm \mu} \pm i e \overline{A_{\mu}} W^{ \pm \mu} \mp i \frac{g \phi}{2 \xi} \phi^{ \pm} & =C^{ \pm}(x), \\
\partial_{\mu} Z^{\mu}-\frac{i}{\xi^{\prime}}\left(g^{2}+g^{\prime 2}\right)^{1 / 2} \phi_{z} & =C_{z},
\end{aligned}
$$


where $e=g \sin \theta_{w}, \tan \theta_{w}=g^{\prime} / g, \phi^{ \pm}, \phi_{z}$ are the Goldstone fields, $\xi, \xi^{\prime}$ are the gauge fixing parameters, $C^{ \pm}, C_{z}$ are arbitrary functions and $\phi_{c}$ is a scalar condensate value. In what follows, all calculations will be done in the general relativistic renormalizable gauge (1),(2) and after that we set $\xi, \xi^{\prime}=0$ choosing the unitary gauge.

To compute the EP $V^{(1)}$ in the background magnetic field let us introduce the proper time, s-representation for the Green functions

$$
G^{a b}=-i \int_{0}^{\infty} d s \exp \left(-i s G^{-1^{a b}}\right)
$$

and apply the method of Ref.[26], allowing in a natural way to incorporate the temperature into this formalism. A basic formula of Ref.26 connecting the Matsubara-Green functions with the Green functions at zero temperature is needed,

$$
G_{k}^{a b}\left(x, x^{\prime} ; T\right)=\sum_{-\infty}^{+\infty}(-1)^{(n+[x]) \sigma_{k}} G_{k}^{a b}\left(x-[x] \beta u, x^{\prime}-n \beta u\right),
$$

where $G_{k}^{a b}$ is the corresponding function at $T=0, \beta=1 / T, u=(0,0,0,1)$, the symbol $[x]$ denotes the integer part of $x_{4} / \beta, \sigma_{k}=1$ in the case of physical fermions and $\sigma_{k}=0$ for boson and ghost fields. The Green functions in the right-hand side of formula (4) are the matrix elements of the operators $G_{k}$ computed in the states $\left.\mid x^{\prime}, a\right)$ at $T=0$, and in the left-hand side the operators are averaged over the states with $T \neq 0$. The corresponding functional spaces $U^{0}$ and $U^{T}$ are different but in the limit of $T \rightarrow 0 U^{T}$ is transformed into $U^{0}$.

The one-loop contribution to the EP is given by the expression [31], [33]

$$
V^{(1)}=-\frac{1}{2} \operatorname{Tr} \log G^{a b}
$$

where $G^{a b}$ stands for the propagators of all the quantum fields $W^{ \pm}, \phi^{ \pm}, \ldots$ in the background magnetic field $H$. In the s-representation the calculation of the trace can be done in accordance with formula 31]

$$
\operatorname{Tr} \log G^{a b}=-\int_{0}^{\infty} \frac{d s}{s} \operatorname{tr} \exp \left(-i s G_{a b}^{-1}\right) .
$$


Details of calculations based on the s-representation and the formula (任) can be found, for example, in Refs. [26], [28]. The terms with $n=0$ in Eqs.(目), (5) give zero temperature contributions to Green's functions and effective potential $V^{(1)}$, respectively. They are the only terms possessing divergences. To eliminate them and uniquely fix the potential we use the following renormalization conditions at $H, T=0[28]$ :

$$
\begin{gathered}
\left.\frac{\partial^{2} V(\phi, H)}{\partial H^{2}}\right|_{H=0, \phi=\delta(0)}=\frac{1}{2}, \\
\left.\frac{\partial V(\phi, H)}{\partial \phi}\right|_{H=0, \phi=\delta(0)}=0, \\
\left.\frac{\partial^{2} V(\phi, H)}{\partial \phi^{2}}\right|_{H=0, \phi=\delta(0)}=\left|m^{2}\right|,
\end{gathered}
$$

where $V(\phi, H)=V^{(0)}+V^{(1)}+\cdots$ is the expansion in the number of loops and $\delta(0)$ is the vacuum value of the scalar field determined in a tree approximation.

It is convenient for what follows to introduce the dimensionless quantities: $h=H / H_{0}\left(H_{0}=M_{w}^{2} / e\right), \phi=\phi_{c} / \delta(0), K=m_{H}^{2} / M_{w}^{2}, B=\beta M_{w}, \tau=1 / B=$ $T / M_{w}, \mathcal{V}=V / H_{0}^{2}$ and $M_{w}=\frac{g}{2} \delta(0)$.

Explicit forms of $V^{(1)}$ at zero temperature are quoted in Refs. [33], [27. Ommiting details of computations which can be found in Refs. [20], [26], [33, we present the finite temperature contributions of boson fields in the form [28]:

$$
\operatorname{Re} \omega_{w}^{(1)}=-4 \frac{\alpha}{\pi} \frac{h}{B}\left(3 \omega_{0}+\omega_{1}-\omega_{2}\right)
$$

where

$$
\begin{gathered}
\omega_{0}=\sum_{p=0}^{\infty} \sum_{n=1}^{\infty} \frac{x_{p}}{n} K_{1}\left(n B x_{p}\right), x_{p}=\left(\phi^{2}+h+2 p h\right)^{1 / 2} \\
\omega_{1}=\sum_{n=1}^{\infty} \frac{y}{n} K_{1}(n B y), y=\left(\phi^{2}-h\right)^{1 / 2}
\end{gathered}
$$

and in the range of parameters $\phi^{2}<h$ after analytic continuation

$$
R e \omega_{1}=-\frac{\pi}{2} \sum_{n=1}^{\infty} \frac{|y|}{n} Y_{1}(n B|y|)
$$




$$
\omega_{2}=\sum_{n=1}^{\infty} \frac{z}{n} K_{1}(n B z), z=\left(\phi^{2}+h\right)^{1 / 2},
$$

and $K_{n}(x), Y_{n}(x)$ are the Bessel functions. The imaginary part of $\omega_{1}$ will be cancelled by the contribution of ring diagrams with the tachyonic mode. So, it is not adduced here.

The fermion finite temperature contribution can be written in the form:

$$
\begin{aligned}
\omega_{f} & =4 \frac{\alpha}{\pi} \sum_{f}\left\{\sum _ { p = 0 } ^ { \infty } \sum _ { n = 1 } ^ { \infty } ( - 1 ) ^ { n } \left[\frac{\left(2 p h+K_{f} \phi^{2}\right)^{1 / 2} h}{B n} K_{1}\left(\left(2 p h+K_{f} \phi^{2}\right)^{1 / 2} B n\right)\right.\right. \\
& \left.\left.+\frac{\left.(2 p+2) h+K_{f} \phi^{2}\right)^{1 / 2}}{B n} h K_{1}\left(\left((2 p+2) h+K_{f} \phi^{2}\right)^{1 / 2} B n\right)\right]\right\}
\end{aligned}
$$

where $K_{f}=m_{f}^{2} / M_{w}^{2}$. The above expressions will be used in the numerical investigations of symmetry behaviour.

3. It was shown by Carrington [25] that at $T \neq 0$ a consistent calculation of the EP based on generalized propagators, which include the polarization operator insertions, requires the ring diagrams to be added simultaneously with the one-loop terms. These diagrams essentially affect the phase transition at high temperatre and zero field [24], 25]. Their importance at $T$ and $H \neq 0$ was also pointed out in literature 20 but, as far as we know, this part of the EP has not been calculated, yet.

As is known [24, the sum of ring diagrams describes a dominant contribution of long distances. It gives significant effect when massless states appear in a system. So, this type of diagrams has to be calculated when a symmetry restoration is investigated. Now, let us turn to computations of $V_{\text {ring }}(H, T)$. It is described by the standard expression [24], 225], 20]:

$$
V_{\text {ring }}=-\frac{1}{12 \pi \beta}\left\{\operatorname{Tr}\left[M^{2}(\phi)+\Pi_{00}(0)\right]^{3 / 2}-M^{3}(\phi)\right\}
$$

where the trace means summation over the all contributing states, $M(\phi)$ is the tree mass of the corresponding state and $\Pi_{00}(0)=\Pi(k=0, T, H)$ for the Higgs particle and $\Pi_{00}(0)=\Pi_{00}(k=0, T, H)$ are the zero-zero components of the polarization operators in a magnetic field taken at zero momenta. The above contribution has order $\sim g^{3}\left(\lambda^{3 / 2}\right)$ in coupling constant whereas the 
two-loop terms are of order $\sim g^{4}, \lambda^{2}$. As $\Pi_{00}(0)$ the high temperature limits of polarization functions have to be substituted which are of orders $\sim T^{2}$ for leading terms and $\sim g \phi_{c} T,(g H)^{1 / 2} T\left(\phi_{c} / T<<1,(g H)^{1 / 2} / T<<1\right)$ for subleading ones.

For the next step of calculations, we remind that the EP is the generating functional of the one-particle irreducible Green functions at zero external momenta. So, to have $\Pi(0)$ we may just calculate the second derivative with respect to $\phi$ of the potential $V^{(1)}(H, T, \phi)$ in the limit of high temperature, $T>>\phi T>>(e H)^{1 / 2}$ and set $\phi=0$. This limit can be calculated by means of the Mellin transformation technique and the result looks as follows:

$$
\begin{aligned}
V^{(1)}(H, & \phi \quad, T \rightarrow \infty)=\left[\left(\frac{C_{f}}{6} \phi_{c}^{2}+\frac{\alpha \pi}{2 \cos ^{2} \theta_{w}} \phi_{c}^{2}+\frac{g^{2}}{16} \phi_{c}^{2}\right) T^{2}\right] \\
& +\left[\frac{\alpha \pi}{6}\left(3 \lambda \phi_{c}^{2}-\delta^{2}(0)\right) T^{2}-\frac{\alpha}{\cos ^{3} \theta} \phi^{3} T-\frac{\alpha}{3}\left(\frac{3 \lambda \phi_{c}^{2}-\delta^{2}(0)}{2}\right)^{3 / 2} T\right] \\
& -\frac{1}{2 \pi}\left(\frac{1}{4} \phi_{c}^{2}+g H\right)^{3 / 2} T+\frac{1}{4 \pi} e H T\left(\frac{1}{4} \phi_{c}^{2}+e H\right)^{1 / 2}+\frac{1}{2} e H T\left(\frac{1}{4} \phi_{c}^{2}-e H\right)^{1 / 2},
\end{aligned}
$$

The parameter $C_{f}=\sum_{i=1}^{3} G_{i l}^{2}+3 \sum_{i=1}^{3} G_{i q}^{2}$ determines the fermion contribution of the order $\sim T^{2}$ having relevance to our problem. We also omitted $\sim$ $T^{4}$ terms in the EP. The terms of the type $\sim \log [T / f(\phi, H)]$ cancel the logarithmic terms in the temperature independent parts. Considering the high temperature limit we restrict ourselves by the linear and quadratic in $T$ terms, only.

Now, differentiating this expression twice with respect to $\phi$ and setting then $\phi=0$, we obtain

$$
\begin{aligned}
\Pi_{\phi}(0) & =\left[\left.\frac{\partial^{2} V^{(1)}(\phi, H, T)}{\partial \phi^{2}}\right|_{\phi=0}\right] \\
& =\frac{1}{24 \beta^{2}}\left(6 \lambda+\frac{6 e^{2}}{\sin ^{2} 2 \theta_{w}}+\frac{3 e^{2}}{\sin ^{2} \theta_{w}}\right) \\
& +\frac{2 \alpha}{\pi} \sum_{f}\left[\frac{\pi^{2} K_{f}}{3 \beta^{2}}-\left|q_{f} H\right| K_{f}\right] \\
& +\frac{(e H)^{1 / 2}}{8 \pi \sin ^{2} \theta_{w} \beta} e^{2}\left(3 \sqrt{2} \zeta\left(-\frac{1}{2}, \frac{1}{2}\right)-1\right) .
\end{aligned}
$$

Here, $K_{f}=m_{f}^{2} / M_{w}^{2}$ and $q_{f}$ is the electric charge of the fermion. We also have added the fermion $\mathrm{H}$-dependent contribution (second term of the fermion 
sum) which eppears in the one-loop EP. The terms $\sim T^{2}$ in Eq.(18) give the known temperature mass squared coming from the boson and fermion sectors. The last $H$-dependent term is negative since the difference in the brackets is $3 \sqrt{2} \zeta\left(-\frac{1}{2}, \frac{1}{2}\right)-1 \simeq-0,39$. Formally, this term has to produce an instability for strong fields but actually it happens for $(e H)^{1 / 2}>>T$ when the asymptotic series is not applicable. Substituting expression (18) into Eq.(16) we obtain (in the dimensionless variables),

$$
\mathcal{V}_{\text {ring }}^{\phi}=-\frac{1}{12 B}\left\{\left(\frac{3 \phi^{2}-1}{2} K+\Pi_{\phi}(0)\right\}^{3 / 2}+\frac{\alpha}{3 B} K^{3 / 2}\left(\frac{3 \phi^{2}-1}{2}\right)^{3 / 2} .\right.
$$

To find the $H$-dependent Debye masses of photons and $Z$-bosons the following procedure will be used. First, we calculate the one-loop EP of the $W$-bosons and fermions in a magnetic field and some "chemical potential", $\mu$, which plays the role of the auxiliary parameter. Then, by differentiating them twice with respect to $\mu$ and setting $\mu=0$ the mass squared $m_{D}^{2}$ will be found. Let us describe that in more detail for the case of fermions where the result is known.

The temperature dependent part of the one-loop EP of constant magnetic field and non-zero chemical potential induced by an electron-positron vacuum polarization is [32]:

$$
V_{\text {ferm. }}^{(1)}=\frac{1}{4 \pi^{2}} \sum_{l-1}^{\infty}(-1)^{l+1} \int_{0}^{\infty} \frac{d s}{s^{3}} \exp \left(\frac{-\beta^{2} l^{2}}{4 s}-m^{2} s\right) e H \operatorname{scoth}(e H s) \cosh (\beta l \mu),
$$

where $m$ is the electron mass, $e=g \sin \theta_{w}$ is electric charge and proper-time representation is used. Its second derivative with respect to $\mu$ taken at $\mu=0$ can be written in the form

$$
\frac{\partial^{2} V_{f e r m}^{(1)}}{\partial \mu^{2}}=\frac{e H}{\pi^{2}} \beta^{2} \frac{\partial}{\beta^{2}} \sum_{l=1}^{\infty}(-1)^{l+1} \int_{0}^{\infty} \frac{d s}{s} \exp \left(-m^{2} s-\beta^{2} l^{2} / 4 s\right) \operatorname{coth}(e H s) .
$$

Expanding coth(eHs) in series and integrating over $s$ we obtain in the limit of $T>>m, T>>(e H)^{1 / 2}$ :

$$
\sum_{l=1}^{\infty}(-1)^{l+1}\left[\frac{8 m}{\beta l} K_{1}(m \beta l)+\frac{2}{3} \frac{(e H)^{2} l \beta}{m} K_{1}(m \beta l)+\cdots\right]
$$

Series in $l$ can easily be calculated by means of the Mellin transformation (see, for example, Refs. [20]). To have the Debye mass squared it is necessary to differentiate Eq.(22) with respect to $\beta^{2}$ and to take into account the 
relation $\mu \rightarrow i e A_{0}$ [20] of the parameter $\mu$ with the zero component of the electromagnetic potential. In this way we obtain finally

$$
m_{D}^{2}=g^{2} \sin ^{2} \theta_{w}\left(\frac{T^{2}}{3}-\frac{1}{2 \pi^{2}} m^{2}+O\left((m \beta)^{2},\left(e H \beta^{2}\right)^{2}\right)\right) .
$$

This is the well known result calculated from the photon polarization operator (see for example 29]). As one can see, the dependence on $H$ appears in the order $\sim T^{-2}$.

Now, let us apply this procedure for the case of the $W$-boson contribution. The corresponding EP (temperature dependent part) calculated at non-zero $T, \mu$ looks as follows,

$V_{w}^{(1)}=-\frac{e H}{8 \pi^{2}} \sum_{l=1}^{\infty} \int_{0}^{\infty} \frac{d s}{s^{2}} \exp \left(-m^{2} s-l^{2} \beta^{2} / 4 s\right)\left[\frac{3}{\sinh (e H s)}+4 \sinh (e H s)\right] \cosh (\beta l \mu)$.

All the notations are obvious. The first term in the squared brackets gives the triple contribution of the charged scalar field and the second one is due to the interaction with the $W$-boson magnetic moment. Again, after differentiation twice with respect to $\mu$ and setting $\mu=0$ it can be written as

$$
\frac{\partial^{2} V_{w}^{(1)}}{\partial \mu^{2}}=\frac{e H}{2 \pi^{2}} \beta^{2} \frac{\partial}{\partial \beta^{2}} \sum_{l=1}^{\infty} \int_{0}^{\infty} \frac{d s}{s} \exp \left(-m^{2} s-\frac{l^{2} \beta^{2}}{4 s}\right)\left[\frac{3}{\sinh (e H s)}+4 \sinh (e H s)\right] .
$$

Expanding $\sinh ^{-1} s$ in series over Bernoulli's polynomials and carrying out all the calculations described above, we obtain for the $W$-boson contribution to $m_{D}^{2}$ of the electromagnetic field,

$$
m_{D}^{2}=3 g^{2} \sin ^{2} \theta_{w}\left[\frac{1}{3} T^{2}-\frac{1}{2 \pi} T\left(m^{2}+g \sin \theta_{w} H\right)^{1 / 2}-\frac{1}{8 \pi^{2}}\left(g \sin \theta_{w} H\right)\right] .
$$

As before, it is necessary to express masses through the vacuum value of the scalar condensate $\phi_{c}$. In the same way the $Z$-boson part $V_{\text {ring }}^{z}$ can be calculated. The only difference is the additional mass term of $Z$-field and an additional term in the Debye mass due to the neutral current $\sim \bar{\nu} \gamma_{\mu} \nu Z_{m u}$. These three fields - $\phi, \gamma, Z$, - which becomes massless in the restored phase, contribute into $V_{\text {ring }}(H, T)$ in an external magnetic field. At zero field there is also a term due to the $W$-boson loops. But when $H \neq 0$ the charged particles acquire $\sim e H$ masses. The corresponding fields remain short-range ones in the restored phase of the vacuum and therefore do not contribute. 
A separate consideration should be spared to the tachyonic (unstable) mode in the $W$-boson spectrum: $p_{0}^{2}=p_{3}^{2}+M_{w}^{2}-e H$. In the fields $e H \sim M_{w}^{2}$ the mode becomes a long range state. Therefore, it has to be included in $V_{\text {ring }}(H, T)$ side by side with other considered neutral fields. But in this case it is impossible to take advantage of formula (16). So, we turn to the EP written in terms of the generalized propagators.

For our purpose it will be convenient to use the expression for the generalized EP written as the sum over the modes in external magnetic field [20]:

$$
V_{\text {gen }}^{(1)}=\frac{e H}{2 \pi \beta} \sum_{l=-\infty}^{+\infty} \int_{-\infty}^{+\infty} \frac{d p_{3}}{2 \pi} \sum_{n=0, \sigma=0, \pm 1}^{\infty} \log \left[\beta^{2}\left(\omega_{l}^{2}+\epsilon_{n, \sigma, p_{3}}^{2}+\Pi(T, H)\right)\right],
$$

where $\omega_{l}=\frac{2 \pi l}{\beta}, \epsilon_{n}^{2}=p_{3}^{2}+M_{w}^{2}+(2 n+1-2 \sigma) e H$ and $\Pi(H, T)$ is the Debye mass of $W$-bosons in a magnetic field. Denoting as $D_{0}^{-1}\left(p_{3}, H . T\right)$ the sum $\omega_{l}^{2}+\epsilon^{2}$, one can rewrite eq. (27) as follows:

$$
\begin{aligned}
V_{\text {gen }}^{(1)} & =\frac{e H}{2 \pi \beta} \sum_{-\infty}^{+\infty} \int_{-\infty}^{+\infty} \frac{d p_{3}}{2 \pi} \sum_{n, \sigma} \log \left[\beta^{2} D_{0}^{-1}\left(p_{3}, H, T\right)\right] \\
& +\frac{e H}{2 \pi \beta} \sum_{-\infty}^{+\infty} \int_{-\infty}^{+\infty} \frac{d p_{3}}{2 \pi}\left\{\log \left[1+\left(\omega_{l}^{2}+p_{3}^{2}+M_{w}^{2}-e H\right)^{-1} \Pi(H, T)\right]\right. \\
& \left.+\sum_{n \neq 0, \sigma \neq+1} \log \left[1+D_{0}\left(\epsilon_{n}^{2}, H, T\right) \Pi(H, T)\right]\right\} .
\end{aligned}
$$

Here, the first term is just the one-loop contribution of $W$-bosons, the second one gives the sum of ring diagrams of the unstable mode ( as it can easily be verified by expanding the logarithm into a series). The last term describes the sum of the short range modes and has to be omitted.

Thus, to find $V_{\text {ring }}^{\text {unstable }}$ one must calculate the second term in Eq. (28). In the high temperature limit we obtain:

$$
V_{\text {ring }}^{\text {unstable }}=\frac{e H}{2 \pi \beta}\left\{\left(M_{w}^{2}-e H+\Pi(H, T)\right)^{1 / 2}-\left(M_{w}^{2}-e H\right)^{1 / 2}\right\} .
$$

By summing up the one-loop EP and all the terms $V_{\text {ring }}$, we arrive at the total consistent in leading order EP.

Let us note the most important featurers of the above expression. It is seen that the last term in Eq.(29) exactly cancels the "dangerous" becoming 
imaginary term in the $V^{(1)}$ Eq. (17). So, no instabilities appear at sufficently high temperatures when $\Pi(H, T)>M_{w}^{2}-e H$ and the EP is real. To make a quantitative estimate of the range of validity of the total EP it is necessary to calculate the mass operator of $W$-boson in a magnetic field at finite temperature and hence to find $\Pi(H, T)$. This is separate and enough complicated problem which is not considered in detail, here. The high temperature limit of the polarization function has been calculated in Ref. 30 and the result looks as follows: $\operatorname{Re} \Pi(H, T)=26,96 \frac{e^{2}}{4 \pi}(e H)^{1 / 2} T$. 円 Below, the obtained mass will be substituted into $V_{\text {ring }}^{\text {unstable }}$ and used in the following estimations.

4. Now, we are going to investigate the symmetry behaviour at high temperature and strong magnetic fields. In order to do that we shall consider the function $\mathcal{V}^{\prime}=\operatorname{Re}[\mathcal{V}(h, \beta, \phi)-\mathcal{V}(h, \beta, 0)]$ describing the symmetry restoration. In fact, we have observed two standard types of that. For weak fields the minimum position of the $\mathrm{EP} \phi_{\min }(h, B)$ is decreased smoothly from unit at low temperature to zero with temperature increasing. That is typical for the second order phase transition. For strong fields $h>0.1$ that corresponds to $H>0.1 \cdot 10^{24} G$ the phase transition becomes of the first order. In this case it is important to check whether the instability threshold $H_{0}=M_{w}^{2} / e$ is desposed in the local minimum of the EP for the field strengths, when the phase transition of the first order happens. That is, whether the effective mass squared $M_{w}^{2}\left(H, T, \phi_{c}(H, T)\right)=\frac{g^{2}}{4} \phi_{c}^{2}\left(H, T_{c}\right)-e H+\Pi\left(H, T_{c}\right)$ is positive or not for the field strengths applied. If this is the case, the calculation is self-consistent and the magnetic field is stable otherwise the evolution of the unstable mode should be considered. The investigation to be of the type as has been carried out in Refs. [8], [9], [34].

Next what is necessary to note is the condition which determines the temperature of the phase transition $T_{c}$. The transition happens for the case

$$
V_{\text {restored }}\left(H, T_{c}, 0\right)=V_{\text {broken }}\left(H, T_{c}, \phi_{c}\left(H, T_{c}\right)\right)
$$

when the depth of the minima located at the begining, $\phi_{c}=0$, and at

\footnotetext{
${ }^{1}$ This result disagrees with the corresponding one of Refs. 13, 13] where this value was found to be zero. Most probably, the discrepancy is because of the calculation procedure used by these authors. They have calculated the polarization operator of the charged gauge field at $H=0$ and then have averaged the result in the state $n=0, \sigma=+1$ calling this "weak field approximation". Our expresion is the high temperature limit of the mass operator which takes into account the external field exactly.
} 
$\phi_{c}=\phi_{\min } \neq 0$ is coinsiding.

In Table 1 we present the results of numeric investigation of the first order phase transition for strong fields $h$. We collected the characteristics of it which are the most important for the baryogenesis problem.

\begin{tabular}{cccccc}
\hline $\mathrm{h}$ & $\mathrm{K}$ & $T_{c}(\mathrm{GeV})$ & $\phi\left(h, T_{c}\right)$ & $R$ & $a$ \\
\hline 1.5 & 0.85 & 105.66 & 0.44385 & 1.03338 & 2.77958 \\
1.5 & 1.25 & 122.36 & 0.38987 & 0.78381 & 2.07133 \\
1.5 & 2 & 146.99 & 0.34496 & 0.57732 & 1.43536 \\
\hline 2 & 0.85 & 105.12 & 0.45717 & 1.06986 & 3.74226 \\
2 & 1.25 & 120.56 & 0.44497 & 0.90795 & 2.84485 \\
2 & 2 & 146.04 & 0.37815 & 0.63698 & 1.92293 \\
\hline
\end{tabular}

\section{Table 1.}

The second column shows the values of the parameter $K=m_{H}^{2} / M_{w}^{2}$, corresponding to the Higgs boson masses $75 \mathrm{GeV}, 90 \mathrm{GeV}$ and $115 \mathrm{GeV}$, respectively. The third one gives the critical temperatures. The fourth column determines the local minimum position (in dimensionless units). It gives possibility to find the mass squared of the W-bosons, $M_{w}^{2}(H, T)=(81 G e V)^{2} \phi^{2}$, and the jump of the order parameter $\phi_{c}\left(H, T_{c}\right)=(246 \mathrm{GeV}) \phi\left(H, T_{c}\right)$. Hence the ratio $R=\phi_{c}(H, T) / T_{c}$ describing the the advantage of the baryogenesis [15] can be calculated. In the last column we show the ratio $a=H / T_{c}^{2}$. It can be used to compare our results with that of other papers.

As one can see, the increase in $h$ makes the phase transition of the first order stronger. However, even for the masses $m_{H} \sim 75 \mathrm{GeV}$ the condition $R>1.2-1.5$ necessary for the baryogenesis [18], [15] is not satisfied. We also note that for strong fields (because of small $\phi(H, T)$ ) the effective W-boson mass squared is negative. That means instability of the local minimum. However, we belive that the presence of the $\mathrm{W}$ - and Z-boson condensates does not increase the $\mathrm{R}$ value. Thus, our analysis shown that for the case of external magnetic field the baryogenesis does not survive in the minimul SM.

Let us compare the results presented with the ones in Refs. [16] - [19]. Remind that these authors have considered the external hypermagnetic field and restricted themselves by its the tree level effects. Besides, they have 
taken into account the contribution of the t-quark, only. Because of these circumstances, they, actually, omitted the most important point that at high temperatures and strong fields the light fermions are important and the local minimum position $\phi_{\min }(H, T)$ is small at the restoring tempereture $T_{c}$. Just the latter point significantly decreases the value of the parameter $R$ in the magnetic field. Since in the broken phase the magnetic and the hypermagnetic fields coincide, our investigation is strightforwardly relevant to the latter case in the respect of the EP curve. Second, in Ref. [17] it was determined that the ratio $a(H, T)=H / T^{2}$ should be of order $\sim 0.1-0.5$. This estimate has then been used in the lattice calculations in Refs. 118, [19. In our calculations for strong magnetic fields the values $a(H, T)$ of larger order have been determined. We would like to complete with other remark concerning the comparison with the hypermagnetic field. Actually, the main mathematical difference between these cases consists in the conditions determining the temperature $T_{c}$. In the hypermagnetic field the condition (30) must be replaced by the one taking into account the partial screening of the external field in the broken phase (see Refs. [16], [17]). Besides, in the restored phase the $\mathrm{W}$-bosond do not interact with the external field, therefore no instabilities occure. We will conside this case in more detail in other publication.

The authors thank Alexei Batrachenko and Vadim Demchik for they help in carring out of numeric calculations.

\section{References}

[1] D. Kirzhnits, JETP Lett. 15 (1972) 529.

[2] A.D. Linde, Particle Physics and Inflationary Cosmology (Harwood Academic, New York, 1990)

[3] M. Sher, Phys. Rep. 179 (1989) 273.

[4] J.R. Espinosa, Preprint DESY 96-107 IEM-FT-96-133, June 1996

[5] T. Vachaspati, Phys. Lett. B265 (1991) 258.

[6] P.H. Brandenberger, A-C. Davis, A.M. Matheson and M. Troden, Phys. Lett. B 293 (1992) 287. 
[7] K. Enqvist, "Magnetic fields in the early universe", astro-ph/9707300 28 July 1997

[8] V.V. Skalozub, Sov. J. Nucl. Phys. 45 (1987) 1058.

[9] J. Ambjörn and P. Olesen, Nucl. Phys. B315 (1989) 606; B330 (1990) 193.

[10] A. Cabo, O.K. Kalashnikov and A. E. Shabad, Nucl. Phys. B 185 (1981) 473.

[11] O.K. Kalashnikov, Fotschr. Phys.. 32325 (1984)

[12] W. Buchmuller and O. Philipsen, Phys. Lett. B397, 112 (1997).

[13] P. Elmfors and D. Persson, Nucl. Phys. B538, 309 (1999), hep$\mathrm{ph} / 9806335$

[14] D. Persson, hep-ph/9901413.

[15] V.A. Rubakov and M. E. Shaposhnikov, CERN-TH/96-13, hepph/9603208 v2 10 Apr 1996.

[16] M. Goivannini and M. Shaposhnikov, Phys. Rev. D57 2186 (1998)

[17] P. Elmfors, K. Enquist and K. Kainulainen, hep-ph/9806403

[18] K. Kajantie, M. Laine, J. Peisa, K. Rummokainen, M. Shaposhnikov, hep-lat/9809004.

[19] M. Laine, hep-ph/9902282.

[20] A.O.Starinets, S.A. Vshivtsev and V.Ch. Zhukovskii, Phys. Lett. B322 (1994) 287.

[21] K. Enqvist and P. Olesen, Phys. Lett. B 329 (1994) 195.

[22] D. Comelli, D. Grasso, M. Pietroni and A. Riotto, The sphaleron in a magnetic field and electrowek phase transition, hep-ph/9903227.

[23] V.V. Skalozub, Int. J. Mod. Phys. A 11 (1996) 5643.

[24] K. Takahashi, Z. Phys. C26 (1985) 601. 
[25] M. Carrington, Phys. Rev. D45 (1992) 2933.

[26] A. Cabo, Fortschr. Phys. 29 (1981) 495.

[27] W. Dittrich, W.-Y. Tsai and K.H. Zimmerman. Phys. Rev. D, 19 (1979) 2929.

[28] Yu.Yu. Reznikov and V.V. Skalozub, Sov. J. Nucl. Phys. 46 (1987) 1085.

[29] A.S. Vshivtsev, V. Ch. Zhukovsky, B.V. Magnitsky and A.V. Tatarintsev, Preprint - 89 -21/ 98 , Inst. Nucl. Phys. Moscow State Univ., Moscow, 1989

[30] V.V. Salozub and A.V. Strel'chenko, In Proceedings of IV Int.Fr eedman Conf., 18-25 June, 1998, St.-Petersburg, Russia (submitt ed for publication in Yadernaya Fizika)

[31] J. Schwinger, Phys. Rev. 82 (1951) 664.

[32] P. Elmfors, D. Persson and B-S. Skagerstam, Phys. Rev. Lett. 71 (1993) 480.

[33] V.V. Skalozub, Sov. J. Part. Nucl. 16 (1985) 445.

[34] S. MacDowell and O. Törnquist, Phys. Rev. D45, (1992) 3833. 